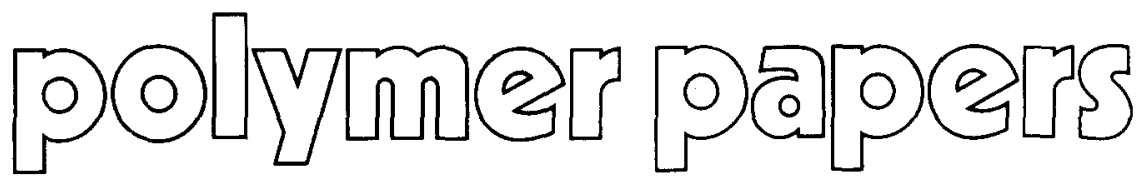

\title{
Multiple scattering in small-angle neutron scattering measurements on polymers*
}

\author{
P. S. Goyal†, J. S. King $\ddagger$ and G. C. Summerfield \\ Department of Nuclear Engineering, The University of Michigan, Ann Arbor, Michigan \\ 48109, USA \\ (Received 8 February 1982)
}

\begin{abstract}
Multiple scattering corrections in small-angle neutron scattering experiments on polymers have been examined. Numerical calculations show that, for typical experimental conditions, the second-order scattering is less than $2 \%$ of the first-order scattering for $Q R_{\mathrm{g}}$ up to 10.0. An approximate expression is also given for obtaining a rough estimate of the second-order scattering without numerical calculation.
\end{abstract}

Keywords Multiple scattering; neutron; small-angle scattering; small-angle neutron scattering; polymers

\section{INTRODUCTION}

Small-angle neutron scattering (SANS) measurements on samples consisting of mixtures of normal and deuterated polymers have been used for a decade to study the configuration of polymer chains in bulk ${ }^{1-6}$ and in concentrated solutions ${ }^{1,7}$. The measured distributions in these experiments contain a finite contribution from neutrons which have suffered more than one scattering event in the sample. However, the effect of the multiply scattered neutrons have not been taken into account in published studies on polymer samples. It has not been certain whether these corrections are indeed small, particularly in experiments using the high concentration tagging technique ${ }^{3-5}$. In this paper, we report a calculation for the relative magnitudes of the secondorder and the first-order scattering in SANS measurements on polymers.

The general problem of multiple scattering in neutron experiments was formulated by Vineyard ${ }^{8}$ in 1954 . This treatment has been used for calculating higher-order scattering events in wide-angle neutron scattering experiments ${ }^{9-12}$. To calculate multiple scattering effects in SANS experiments, Schelten and Schmatz ${ }^{13}$ suggested a somewhat different procedure, which involves numerical evaluation of the Fourier transforms of the scattering functions. This was used for neutron scattering from spheres ${ }^{13}$ where the coherent scattering function $\Sigma_{\text {coh }}(Q)$ varies rapidly $\left(Q^{-4}\right)$ at large wavevector transfer $Q$. We have found that in the case of polymer chains, where $\Sigma_{\text {coh }}(Q)$ varies as $Q^{-2}$ at large $Q$, the Fourier transform method results in significant truncation errors for practical limits of integration. We have, therefore, used Vineyard's formulation for the present calculations. The theory of the method is given in the next section. Results and discussion of the calculations are given in the third section. The fourth section gives an analytical expression

* Work supported by National Science Foundation Grant No. DMR$79-26254$

+ On leave from Nuclear Physics Division, Bhabha Atomic Research Centre, Bombay 400085, India

$\ddagger$ To whom correspondence should be addressed for obtaining a crude estimate of the second-order scattering. We summarize these results in the final section.

\section{THEORY}

We consider neutron scattering from an infinite slab of thickness $t$. A well collimated beam of neutrons of wavevector $\mathbf{k}_{0}$ and a uniform current density $I_{0}$ neutrons per square centimetre per second per steradian is incident along the normal to the flat surface of the sample. $k$ is the wavevector of the neutrons that emerge from the sample and proceed towards the detector. $k$ makes an angle $\theta$ with the slab normal as shown in Figure 1. In general, the neutrons might be scattered in the specimen several times

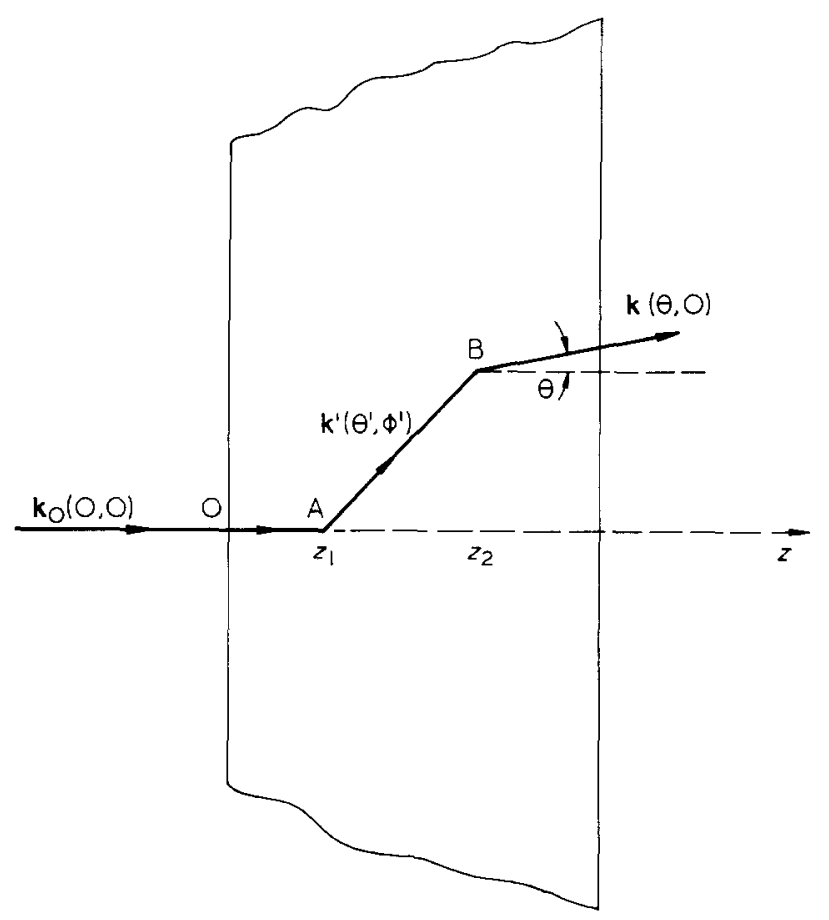

Figure 1 Scattering geometry for the two scattering situation. $\boldsymbol{k}_{0}$ $\boldsymbol{k}$ and the slab normal are in $\phi=0$ plane. Direction of $\boldsymbol{k}^{\prime}$ is given by polar angles $\left(\theta^{\prime}, \phi^{\prime}\right)$ 
before they emerge. In the following, we obtain the expression for the second-order scattered neutron current $I_{2}(Q)$ as a function of the wavevector transfer $Q\left(=\boldsymbol{k}_{0}-\boldsymbol{k}\right)$ using an approach similar to that developed by Vineyard ${ }^{8}$ and later workers ${ }^{9-12}$.

Let us assume that the two scattering events occur at $A$ and $\mathrm{B}$ respectively (Figure 1 ). The incident neutron is first elastically scattered at A and proceeds along $\boldsymbol{k}^{\prime}$. It suffers a second elastic scattering at $\mathbf{B}$ and emerges from the sample in the direction of $\boldsymbol{k}$. To describe the trajectory of the neutron, we use a spherical coordinate system with its pole along the inward slab normal. The polar angles $\left(\theta^{\prime}\right.$, $\left.\phi^{\prime}\right)$ and $(\theta, \phi)$ give the directions of $\boldsymbol{k}^{\prime}$ and $\boldsymbol{k}$ respectively. We assume that the incident beam is well collimated such that $\boldsymbol{k}_{0}$ has angles $(0,0)$. We assume $I_{2}(Q)$ along $(\theta, \phi)$ to be independent of the azimuthal angle $\phi$. It is thus convenient to choose $\boldsymbol{k}_{0}, \boldsymbol{k}$ and the slab normal to be coplanar and to measure the azimuthal angle $\phi$ from this plane. That is, we assume $\phi=0$. If $A$ and $B$ are situated at depths $z_{1}$ and $z_{2}$ from the flat surface of the sample and if one assumes that both of the scatterings are forwards $\left(\theta, \theta^{\prime}\right.$ $<\pi / 2$ ), then it can be shown ${ }^{8,12}$ that

$$
\begin{aligned}
I_{2}(Q)= & I_{0} \int_{0}^{\pi / 2} d \theta^{\prime} \sin \theta^{\prime} \int_{0}^{2 \pi} \mathrm{d} \varphi^{\prime}\left(\int_{0}^{t} \mathrm{~d} z_{1} \sec \theta^{\prime} \mathrm{e}^{-\mu z_{1}} \Sigma\left(\theta^{\prime}\right)\right) \\
& \left.\times \int_{=1}^{t} \mathrm{~d} z_{2} \sec \theta \mathrm{e}^{-\mu\left(z_{2}-z_{1}\right) \sec \theta^{\prime}} \Sigma(\beta) \mathrm{e}^{-\mu(t-z) \sec \theta}\right)
\end{aligned}
$$

Here $\mu$ is the attenuation coefficient which includes absorption and all scattering processes including smallangle coherent scattering (SACS). $\Sigma(\theta)$ is the macroscopic differential scattering cross-section for the neutrons to be scattered through an angle $\theta$. In the present problem, the scattering angle at first scattering is $\theta^{\prime}$ and that at second scattering is $\beta$ such that

$$
\cos \beta=\cos \theta^{\prime} \cos \theta-\sin \theta^{\prime} \sin \theta \cos \phi^{\prime}
$$

Equation (1) can be simplified to obtain

$$
I_{2}(Q)=2 I_{0} \mathrm{e}^{-\mu t} \int_{0}^{\pi / 2} \Sigma\left(\theta^{\prime}\right) f_{2}\left(\theta, \theta^{\prime}\right) \sin \theta^{\prime} \mathrm{d} \theta^{\prime} \int_{0}^{\pi} \Sigma(\beta) \mathrm{d} \varphi^{\prime}
$$

where

$$
f_{2}\left(\theta, \theta^{\prime}\right)=\frac{\sec \theta \sec \theta^{\prime}}{\mu\left(\sec \theta^{\prime}-\sec \theta\right)}\left(\frac{\mathrm{e}^{-\mu t\left(\sec \theta^{\prime}-1\right)}-1}{\mu\left(\sec \theta^{\prime}-1\right)}-\frac{\mathrm{e}^{-\mu t(\sec \theta-1)}-1}{\mu(\sec \theta-1)}\right)
$$

It can be shown in a similar way that the first-order current $I_{1}(Q)$ emerging from the sample in the direction of $\boldsymbol{k}$ is given by

$$
I_{1}(Q)=I_{0} \mathrm{e}^{-\mu t} \Sigma(\theta) f_{1}(\theta)
$$

where

$$
f_{1}(\theta)=\frac{\sec \theta\left(1-\mathrm{e}^{-\mu i \sec \theta-1)}\right.}{\mu(\sec \theta-1)}
$$

In SANS experiments on polymers, neutron scattering is dominated by SACS events. In general, there may also be a small contribution to $\Sigma(\theta)$ from incoherent scattering; we discuss this effect in the next section. For the present, we assume $\Sigma(\theta)=\Sigma_{\text {coh }}(\theta)$ only. This is determined by the configuration of the polymer chain and by the overall density fluctuations in the sample ${ }^{3}$.

\section{CALCULATION DETAILS AND RESULTS}

It has been seen from equation (3) that, to calculate the second-order scattering corrections, one has to know $\Sigma_{\text {coh }}(\theta)$, the quantity one wants to measure. In principle, one can obtain $\Sigma_{\text {coh }}(\theta)$ and $I_{2}(Q)$ from the measured distribution using an iterative procedure if the higherorder scatterings are negligible. This requires a knowledge of $\Sigma_{\mathrm{coh}}(\theta)$ over a large region of $\theta$. In general, the measured distributions are not accurate at large $\theta$. In view of this, we calculate instead the relative magnitudes of $I_{2}(Q)$ and $I_{1}(Q)$ using a model for the scattering function $\Sigma_{\mathrm{coh}}(\theta)$. For the present purpose, we shall assume the polymer chains to be Gaussian (Debye model ${ }^{14}$ ). The effect of the density fluctuations on $\Sigma_{\text {coh }}(\theta)$ is normally small for bulk amorphous polymers, particularly for bulk polystyrene ${ }^{4}$; and we neglect this contribution. Then for a mixture of normal and deuterated polymer, it can be shown ${ }^{3}$ that

$$
\Sigma_{\text {coh }}(\theta)=\frac{2 C}{p^{2}}\left(p-1+\mathrm{e}^{-p}\right)
$$

where

$$
C=\left(a_{\mathrm{H}}-a_{\mathrm{D}}\right)^{2} N n^{2} \chi(1-\chi)
$$

and

$$
p=\left(2 k_{0} \sin \frac{1}{2} \theta\right)^{2} R_{g}^{2}=Q^{2} R_{g}^{2}
$$

Here $R_{g}$ is the radius of gyration for the polymer chain. $a_{\mathrm{H}}$ and $a_{\mathrm{D}}$ are the coherent scattering lengths per protonated and deuterated monomer respectively. $N$ is the number of polymer chains per unit volume of the sample and $n$ is the number of monomers in each chain. $\chi$ is the number fraction of monomers that are deuterated in the sample. We use this model for all coherent elastic scattering events. While equation (7) is incorrect for large $Q$, and hence large scattering angles, the error is made negligible by the rapid reduction in $\Sigma_{\text {coh }}(\theta)$ as $\theta$ increases.

The first- and second-order scatterings $I_{1}(Q)$ and $I_{2}(Q)$ have been numerically computed using equations (3)-(7) for bulk polystyrene samples of molecular weight $M_{w}$ $=194000, t=0.12 \mathrm{~cm}$ and $\chi=0.3$ corresponding to typical high concentration sample conditions ${ }^{4} . R_{g}$ for a polystyrene chain of molecular weight $M_{w}$ is equal to $M_{w}^{1 / 2}$ times a well established constant ${ }^{2,4,6}$. That is

$$
R_{g}=0.267 M_{w}^{1 / 2}
$$

$k_{0}$ was taken to be $1.323 \AA^{-1}$. The integrations in equation (3) were carried out numerically on the $\theta^{\prime}$ range $\left[0, \theta_{\max }^{\prime}\right]$ and simultaneously in the $\phi^{\prime}$ range $[0, \pi]$. The restriction on $\theta_{\max }^{\prime}$ shortens the computation time and introduces only an insignificant error because $\Sigma_{\text {coh }}\left(\theta^{\prime}\right)$ becomes negligibly small at large angles. Calculations for $I_{2}(Q)$ have been made for $\theta_{\max }^{\prime}$ varying from $\pi / 25$ up to 


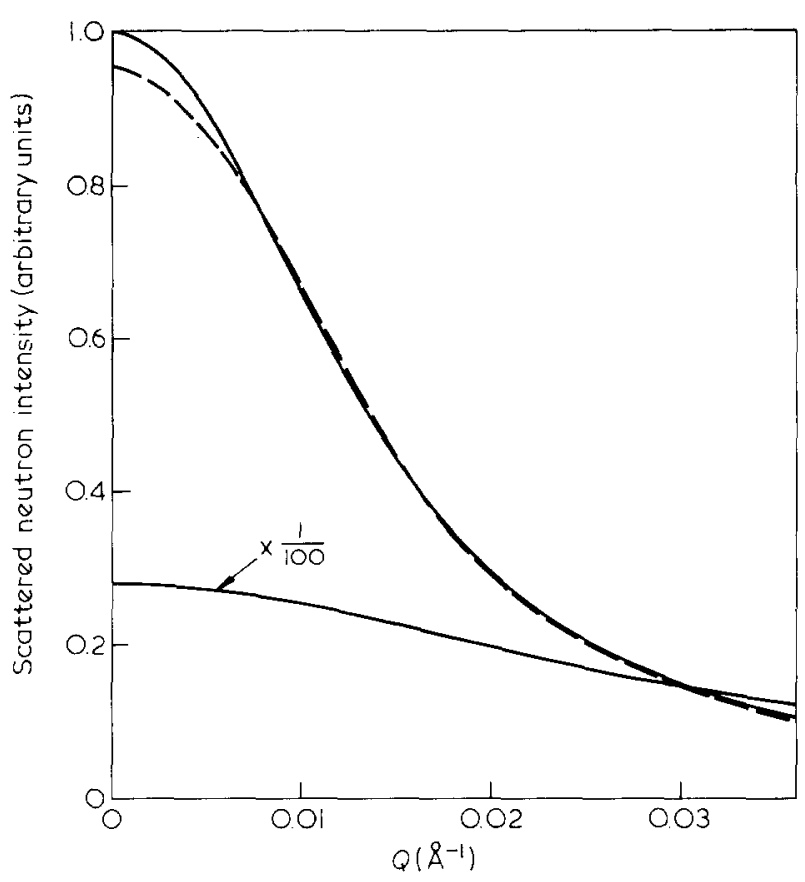

Figure 2 Scattered neutron intensity versus $Q$ for the Debye chains. The upper and lower full curves are numerically calculated $I_{1}(Q)$ and $I_{2}(Q)$ using Vineyard's method as discussed in the text. The broken curve is the tota/ scattering $I(Q)$ calculated using the method of Schelten and Schmatz

$\pi / 10$ and show constant results within $2 \%$. The results shown in Figure 2 correspond to $\theta_{\max }^{\prime}=\pi / 15$. In this figure, the upper and lower full curves correspond to $I_{1}(Q)$ and $I_{2}(Q)$ respectively (note a factor $1 / 100$ for $I_{2}(Q)$ ). This gives a ratio of $I_{2}(Q) / I_{1}(Q)$ in the range of about 0.28 to $1.2 \%$. In view of this result, the third-order scattering is expected to be negligible ${ }^{8}$.

In these calculations, we have assumed the neutron scattering to be purely coherent. Incoherent scattering is not negligible for a sample consisting of a mixture of normal and deuterated polymers. While $\Sigma_{\text {coh }}(\theta)$ decreases with increase in $\theta$, the incoherent scattering cross-section $\Sigma_{\text {inc }}(\theta)$ remains approximately constant. For the experimental situation under discussion, $\Sigma_{\text {inc }}(0) / \Sigma_{\text {coh }}(0)$ $\simeq 0.005$ in the forward direction and $\Sigma_{\text {inc }}(\theta)$ is larger than $\Sigma_{c i h}(\theta)$ for $\theta>\pi / 15$. In view of this, one expects the total second-order scattering $I_{2}^{\mathrm{t}}(Q)$ to consist of three parts $I_{2}^{\mathrm{a}}(Q), \quad I_{2}^{\mathrm{b}}(Q)$ and $I_{2}^{\mathrm{c}}(Q)$ arising from three types of scattering events: (a) two coherent scattering events, (b) one coherent and one incoherent scattering event, and (c) two incoherent scattering events. The second-order scattering curve shown in Figure 2 corresponds to $I_{2}^{\text {a }}(Q)$. Calculations similar to the one described above were carried out using

$$
\Sigma(\theta)=\Sigma_{\mathrm{coh}}(\theta)+\Sigma_{\text {inc }}(\theta)
$$

These calculations gave a second-order intensity which was about $5 \%$ larger than that shown in Figure 2. We believe this extra intensity corresponds to $I_{2}^{\mathrm{b}}(Q)$. We need not calculate $I_{2} \mathrm{c}(Q)$ as it is removed by the experimental procedure of subtracting the scattering from a control sample from the total scattering of the marked sample. This approximately corrects the data for multiple incoherent scattering also. In view of the above discussion, the effect of the incoherent scattering on the present calculations is ignored. We assume $I_{2}^{\mathrm{a}}(Q)=$ $I_{2}^{\mathrm{t}}(Q)=I_{2}(Q)$. It is seen that multiple scattering corrections are quite small. The radius of gyration $R_{\mathrm{g}}$ obtained from the uncorrected data is expected to be accurate to $1 \%$.

It should be noted that if the same problem is tested using the method of Schelten and Schmatz ${ }^{13}$, the secondorder scattering term $I_{2}(Q)$ is comparable to within $5 \%$ of that shown in Figure 2. However, the total scattering

$$
I(Q)=\sum_{n=1}^{x} I_{n}(Q)
$$

is significantly different as shown by the broken curve of Figure 2 and leads to an apparent change in $R_{\mathrm{g}}$ of about $10 \%$. This is because $I(Q)$ suffers from large truncation errors even when the limits of integration in the numerical evaluation of the Fourier transforms were taken to be 1.5 times larger than those used by Schelten and Schmatz. Oscillations in the calculated $I(Q)$ were also seen. We believe this is because of the slow $\left(Q^{-2}\right)$ decrease in $\Sigma_{\text {coh }}(Q)$ with $Q$ for the polymer chains. The Schelten and Schmatz calculation is for spheres and because of the $Q^{-4}$ dependence does not suffer from truncation errors.

\section{ANALYTICAL EXPRESSION FOR SECOND- ORDER SCATTERING}

An analytical expression for the second-order scattering $I_{2}(Q)$ has been obtained following the treatment of Schelten and Schmatz but approximating the true form of $\Sigma_{\mathrm{coh}}(Q)$ by a Lorentzian function of the form

$$
\Sigma_{\mathrm{coh}}(Q)=\frac{C}{1+Q^{2} R_{\mathrm{g}}^{2 / 3}}
$$

This is a reasonable approximation to the Debye equation (7). Schelten and Schmatz showed that

$$
I_{n}(Q)=\frac{I_{0}}{2 \pi} \int_{0}^{x} J_{0}(Q r) h_{n}(r) r \mathrm{~d} r
$$

where

$$
h_{n}(r)=\frac{k_{0}^{2}}{n !}\left(\frac{s(r)}{k_{0}^{2}}\right)^{n} \mathrm{e}^{-\mu t}
$$

and $J_{0}(Q r)$ is a Bessel function of zero order. $s(r)$ in the above expression is given by

$$
s(r)=2 \pi \int_{0}^{\infty} J_{0}(Q r) \Sigma_{\mathrm{coh}}(Q) t Q \mathrm{~d} Q
$$

Using equations (8), (9) and (10), it can be shown that

$$
I_{2}(Q)=\frac{9 \pi I_{0} C^{2} t^{2} \mathrm{e}^{-\mu t}}{R_{\mathrm{g}}^{2} k_{0}^{2}} F\left(Q R_{\mathrm{g}}\right)
$$

where

$$
F\left(Q R_{\mathrm{g}}\right)=F(p)=\frac{1}{p^{1 / 2}(12+p)^{1 / 2}} \ln \left(\frac{(12+p)^{1 / 2}+p^{1 / 2}}{(12+p)^{1 / 2}-p^{1 / 2}}\right)
$$




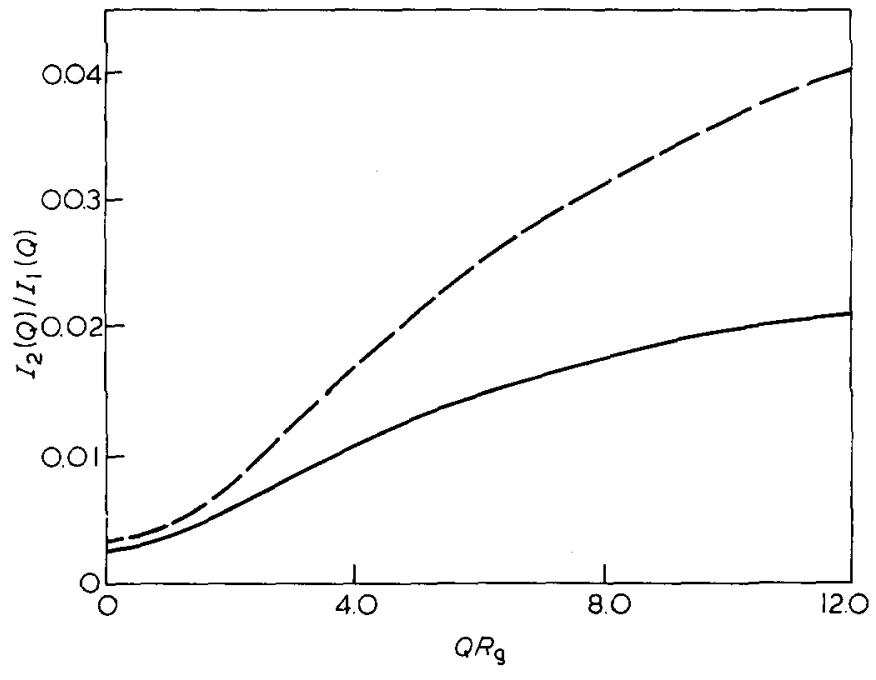

Figure $3 I_{2}(Q) / I_{1}(Q)$ as a function of $Q R_{\mathrm{g}}$. For the full curve, $I_{2}(Q)$ is that for the Debye chains and was obtained numerically using equation ( 3$)$. For the broken curve, $I_{2}(Q)$ was obtained from the analytical expression (11). For both curves $I_{1}(Q)$ was obtained using equation (7) (Debye model)

A comparison of this expression* with the numerical results of the previous section is shown in Figure 3 for $M_{w}$ $=10^{6}$. The broken and full curves correspond to analytical and numerical results, respectively. Here, instead of $I_{2}(Q)$, we have plotted $I_{2}(Q) / I_{1}(Q)$ as a function of $Q R_{\mathrm{a}}$ - and have used the same $I_{1}(Q)$ (equation (7)) for both the curves. It is seen that compared to the numerical results equation (11) gives 25 to $90 \%$ higher $I_{2}(Q)$. The curve is also valid for lower molecular weights and can be used for estimating the relative magnitudes of the secondand first-order scatterings for polymer samples.

\section{SUMMARY}

The relative magnitudes of the second-order and the firstorder scatterings in SANS experiments on mixtures of

* Equation (11) gives $I_{2}(Q)$ identical to the one obtained numerically using equation (3) with a Lorentzian function for $\Sigma_{\mathrm{coh}}(Q)$. normal and deuterated polymers have been calculated numerically using Vineyard's method. The calculations for $0.12 \mathrm{~cm}$ thick samples of bulk polystyrene, containing $30 \%$ deuterated chains, show that the second-order scattering is less than $2 \%$ of the first-order scattering up to $Q R_{\mathrm{g}}=10.0$. The second-order scattering is expected to be smaller when the concentration of the tagged chains is smaller. Consequently, we believe that the multiple scattering corrections in SANS experiments on polymers are normally quite small.

An approximate expression for obtaining the secondorder scattering is also given. A comparison of this expression with the numerical results shows that it overestimates the second-order scattering. This expression may be considered a conservative upper limit of the second-order scattering in SANS experiments on polymers and does not require numerical calculation.

\section{REFERENCES}

1 Higgins, J. S. in 'Treatise in Materials Science and Technology', Vol. 15 (Ed. G. Kostorz), Academic Press, New York, 1979, and references therein

2 Cotton, J. P., Decker, D., Benoit, H., Farnoux, B., Higgins, J., Jannink, G., Ober, R., Picot, C. and des Cloizeaux, J. Macromolecules 1974, 7, 863

3 Akcasu, Z. A., Summerfield, G. C., Jahshan, S. N., Han, C. C., Kim, C. Y. and Yu, J. J. Polym. Sci., Polym. Phys. Edn. 1980, 18, 863

4 Tangari, C., Summerfield, G. C., King, J. S., Berliner, R. and Mildner, D. F. R. Macromolecules 1980, 13, 1546

5 Wignall, G. D., Hendricks, R. W., Koehler, W. C., Lin, J. S., Wai, M. P., Thomas, M. L. and Stein, R. S. Polymer 1981, 22, 886

6 Tangari, C., King, J. S. and Summerfield, G. C. Macromolecules $1982,15,132$

7 Daoud, M., Cotton, J. P., Farnoux, B., Jannink, G., Sarma, G., Benoit, H., Dupplessix, R., Picot, C. and de Gennes, P. G. Macromolecules 1975, 8, 804

8 Vineyard, G. H. Phys. Rev. 1954, 96, 93

9 Blech, I. A. and Averbach, B. L. Phys. Rev. A 1965, 137, 1113

9 Blech, I. A. and Averbach, B. L. Phys. Rev.
10 Cocking, S. J. AERE Rep. 5867, 1968, p. 1

11 Rao, K. R., Dasannacharya, B. A. and Yip, S. J. Phys. C, Solid St. Phys. 1971, 4, 2725

12 Agrawal, A. K. Phys. Rev. A 1971, 4, 1560

13 Schelten, J. and Schmatz, W. J. Appl. Crystallogr. 1980, 13, 385

14 Debye, P. J. Phys. Colloid. Chem. 1947, 51, 18 